Original Article

\title{
A modified point count method as a practical approach to assess the tumor volume and the percent gland involvement by prostate carcinoma
}

\author{
Paulo Roberto Fontes Athanazio ${ }^{\mathrm{a}, \mathrm{b}}$, Andréia Carvalho dos Santos ${ }^{\mathrm{a}, \mathrm{c}}$, \\ Luiz Antonio Rodrigues de Freitas ${ }^{a, b, c}$, Daniel Abensur Athanazio ${ }^{\text {b,c,* }}$ \\ a IMAGEPAT Laboratory of Pathology, Rua Lucaia, $n^{\circ}$ 209, Salvador, Brazil \\ ${ }^{\mathrm{b}}$ Department of Pathology, Largo do Terreiro de Jesus s/n, Federal University of Bahia, Salvador, Brazil \\ c Gonçalo Moniz Research Center, Rua Waldemar Falcão, 121, Oswaldo Cruz Foundation, Salvador, Brazil
}

\section{A R T I C L E I N F O}

\section{Article history:}

Received 11 December 2013

Received in revised form 25 January 2014

Accepted 10 February 2014

\section{Keywords:}

Prostatic neoplasms

Pathology

Prostate-specific antigen

Tumor burden

\begin{abstract}
A B S T R A C T
This study reports a modified point-count method for quantifying the extent of carcinoma in prostatectomy specimens ( $n=143)$, as adapted from Billis et al. (2003) [3]. The prostates were studied as follows: the basal/apical margins were sampled using the cone method. The remainder of the gland was divided into 12 quadrant-shaped regions that were sampled using two slices. Eight equidistant points were marked directly on the coverslip over each fragment. The points inside the tumoral areas were counted and expressed as both the percentage of prostate gland involvement by carcinoma (PGI) and the tumor volume (TV). A significant correlation between the preoperative PSA levels and each of the three quantitative estimations were observed, with improved correlations with the PGI and TV values obtained using the point-count method (viz. number of slices involved (NSI) $(r=0.32)$, PGI $(r=0.39)$ and TV $(r=0.44)$ ). With the data sets stratified into three categories, all three methods correlated with multiple parameters, including Gleason scores $\geq 7$, primary Gleason scores $\geq 4$, perineural/angiolymphatic invasion, extraprostatic extension, seminal vesicle invasion and positive margins. All three quantitative methods were associated with morphologic features of tumor progression. The results obtained using this modified point-count method correlate more strongly with preoperative PSA levels.
\end{abstract}

(C) 2014 Elsevier GmbH. All rights reserved.

\section{Introduction}

Prostate carcinoma has a high incidence in developed countries, with more than 643,000 new cases in 2008 , representing $20 \%$ of all malignancies in males. Compared with the $70 \%$ ten-year survival rate observed by patients in developed countries, mortality rates from prostate carcinoma are asymmetrically higher in developing regions of the world [32]. In Brazil, more than 60,000 new cases were diagnosed in 2012, reflecting an annual incidence of $62 / 100,000$ [14]. The College of American Pathologists (CAP) classifies prognostic factors in categories I (proven prognostic value and useful in daily practice), II (factors supported by preliminary

\footnotetext{
* Corresponding author at: Universidade Federal da Bahia, Faculdade de Medicina da Bahia, Departamento de Patologia e Medicina Legal, Praça XV de novembro, s/n Largo do Terreiro de Jesus, 40025010 Salvador, BA, Brazil. Tel.: +55 71 32835563x41; fax: +55 7132408406 .

E-mail addresses: daa@ufba.br, dathanazio@gmail.com (D.A. Athanazio).
}

experimental and clinical data, but waiting validation in large clinical studies) and III (other suggested factors). The category I prognostic factors for prostate carcinoma include pre-operative serum prostatic specific antigen (PSA) levels, Gleason grade, staging and surgical margin involvement at prostatectomy. Upon completion of the examination of a prostatectomy specimen, the relevant morphologic findings that are consequential in guiding patient treatments and follow up include the Gleason grade, extraprostatic extension, seminal vesicle invasion, positive surgical margins, intraprostatic perineural invasion, tumor volume and nodal metastasis [7].

According to a survey by the American Society of Clinical Pathologists, only $12 \%$ of pathologists use total embedding as the routine method for the examination of radical prostatectomy specimens [28]. In a recent survey, the International Society of Urologic Pathology (ISUP) made efforts to reach minimal consensus on the handling of prostatectomy specimens. Several widespread practices, such as inking margins and the cone method for the examination of both apex and base, were included in the consensus determination. 
Both the total embedding of the prostate and the use of whole mounted sections (to assure the identification of the diameter of the largest tumor focus) were not included in this consensus [23]. In the last ISUP survey, the consensus determination suggested that the pathology report of a prostatectomy specimen should include a quantitative estimate of the cancer volume, with the specific protocol decided by each laboratory. As part of this recommendation, this protocol should be well-defined and standardized for the appropriate use by all pathologists within the institution [30]. Several experts, however, explicitly recommend a reporting of the percentage of tumor involvement in all prostatic tissue specimens $[12,26]$. The report from the consensus conferences of ISUP recognized that a unified method to measure and report the cancer volume from a radical prostatectomy has long been a controversial issue in urologic pathology, with considerable debate on the magnitude of effort that pathologists should invest in determining the cancer volume values to be included in the pathology reports.

Tumor volume has been previously associated with decreased survival and has been included in the morphologic criteria for poor prognoses, including high grade and advanced stage diagnoses $[11,13,18,20,21]$. Many studies on this issue have failed to support these observations $[25,30]$. Several studies have been limited by the inclusion of locally advanced disease cases, while both tumor size and percentage are considered to be important measures in particular for those patients with organ confined tumors [10]. Large tumors may display indolent behaviors, remaining confined in a large prostate with concomitant nodular hyperplasia. Several studies have shown that the percentage of the gland involved by the carcinoma has prognostic value $[4,17,20,22]$, with this factor more predictive than the tumor volume $[17,20]$. Recently, several authors have suggested the use of the diameter of the largest tumor focus as a morphologic feature with prognostic value $[6,8]$. The estimation of this factor would most likely require the use of whole mounted sections, which is the current practice used by $16 \%$ of surveyed uropathologists [23].

The authors of this present study recognize that a major step in the advancement to develop the method to quantify and report the extent of the tumor in prostatectomy specimens was presented in the proposal by Billis and colleagues [2,3]. This advancement in the protocol included several elements: (1) the basal (bladder neck) and apical margins were sectioned into cones and cut into sagittal slices; (2) the serial transverse sections of the specimen were further sliced into quadrants; (3) the tumor areas were contoured in the hematoxylin-eosin glass slides; (4) the contoured areas were manually transferred onto a sheet of paper (drawn) using quadrants containing eight equidistant points; (5) the total amount of positive points (inside the countered areas) represented an estimation of the tumor extent. This method has been adopted as a recommended method to handle radical prostatectomy specimens by the Brazilian Society of Pathology [1]. Even with the simplicity and practicality of this protocol (avoiding computer based morphometry, for instance), the point count method has not been widely adopted. The authors of this present study suggest that there are several disadvantages in the original proposal:

- transferring the drawings from glass slides to sheets of paper may not be attractive for most pathologists, as this method is time consuming;

- the pathologist who draws in the points within the quadrants may be influenced by previous observations of the points;

- the number of quadrants examined in each specimen can be variable (12-56), possibly as result of a method of obligatory total embedding;

- the results are expressed as a number of positive points, which is not an estimate that is easily understood by urologists and oncologists. Other parameters, such as either the percentage of

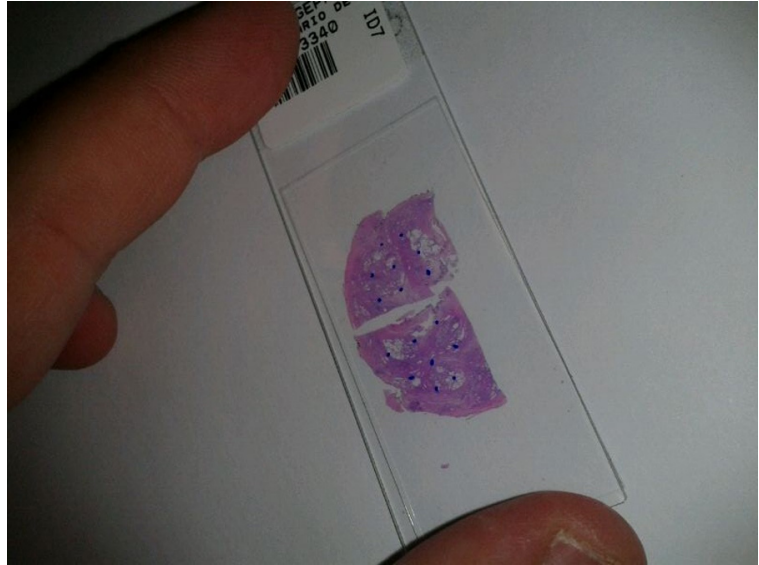

Fig. 1. The point count method. Each hematoxylin-eosin stained glass slide (containing two slices) had eight equidistant points painted directly over the fragments.

prostate involvement by the carcinoma or the tumor volume, are universal with an obvious interpretation in a pathology report.

In this present report, an alternative point count method is proposed that provides improved simplicity, allowing an expression of the results as a direct estimate of either the percentage of prostate involvement by the carcinoma or the tumor volume.

\section{Materials and methods}

\section{Patients and protocols}

All consecutive radical prostatectomy specimens examined in the Laboratory of Pathology IMAGEPAT (Salvador, Brazil) from May 2010 (the date that the proposed protocol was adopted) to May 2013 were included in the study. The project was approved by the Research Ethics Committee of Centro de Pesquisas Gonçalo Moniz (CPqGM/FIOCRUZ) located in Salvador, Brazil.

All radical prostatectomy specimens were examined using the same protocol. For each specimen, the weight, measurement and volume were documented. The entire external surface was inked. Both the apical and basal margins were sectioned according to the cone method and sliced in the sagittal plane. Two sections of the proximal third of each seminal vesicle and deferent duct were processed. The remnant prostate was transversally sectioned into three thirds of equivalent thickness, representing an apical, an intermediate and a basal third. Each region was sliced into quadrants (resulting in twelve zones). Each zone was further sectioned, alternating one slice for the gross specimen archive and one slice to be processed. Two slices were processed for each region, alternating one slice (24 slices in the quadrant shape, including the circumferential margin). Each resultant hematoxylin-eosin stained glass slide (containing two slices) had eight equidistant points painted directly over the fragments (Fig. 1). The points were inserted in the same manner used in the quadrants on the sheets of paper from the original point count protocol [3]. After a microscopic examination, points that were inside the tumor area were counted. The point count was recorded from the slice with more involved points in each one of the twelve sampled regions. The microscopic report included the number of slices involved ( $x$ of 2 ) and the points involved ( $x$ of 8 ) for each one of the 12 different regions. As the number of possible points to be counted was 96 , the total point count was used to approximate the percentage of gland involvement by the prostate carcinoma. The conversion of the percentage of gland involvement in the tumor volume was based on an assumption that the area fraction of a phenomenon in a solid is equivalent 


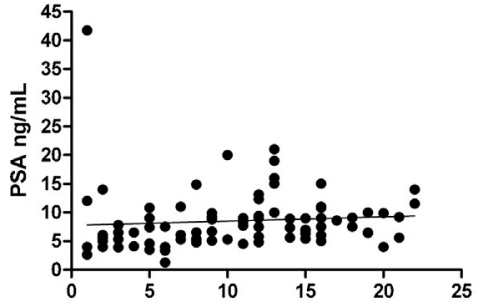

A

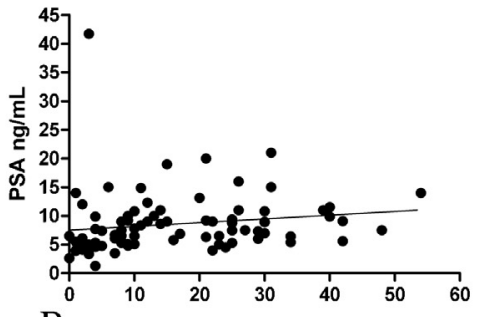

B

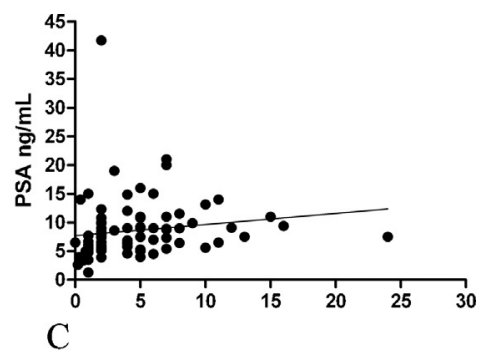

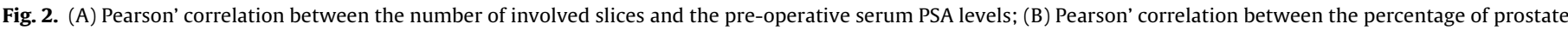
involvement and the pre-operative serum PSA levels; (C) Pearson' correlation between the tumor volume and the pre-operative serum PSA levels.

to the volume fraction of that phenomenon. The tumor volume was calculated from the total volume of the prostate multiplied by the proportion of the gland involved by the cancer [31].

Two estimates of the tumor extent based on the point count method (percentage of gland involvement (PGI) and tumor volume) and the more widely used number of slices involved (NSI) were evaluated for possible correlations with the pre-operative levels of total prostate specific antigen (PSA) and multiple morphologic parameters of prognosis that are routinely reported in radical prostatectomy specimens, including histologic grade (Gleason score and primary Gleason), intraprostatic perineural invasion, angiolymphatic invasion, extraprostatic extension, circumferential margins, apical and basal margins, microscopic invasion of bladder neck and seminal vesicle invasion.

\section{Statistical analyses}

Statistical analyses were performed using the GraphPad Prism 4.03 software package (Graph Pad, CA, USA). Categorical data were compared by a Chi-square test for trend, and the numerical data were compared using a student's $t$-test.

\section{Results}

A total of 143 radical prostatectomy specimens were examined during the study period. One case $(0.7 \%)$ had no tumor in the initial 24 slides and required a total embedding process for the detection of a small cancer focus in one of 144 slices. As shown in Fig. 2, all three measurements exhibited a significant moderate positive correlation with pre-operative PSA levels, with improved correlations for both values (PGI and TV) obtained using the point-count method (viz.: NSI $(r=0.32)$, PGI $(r=0.39)$ and tumor volume $(r=0.44))$.

For the measured values, the means of the tumor extent stratified by cases either with or without the ten morphologic features with prognostic value that are routinely reported in radical prostatectomy specimens were determined and shown in Table 1. Among the parameters examined, the PGI was significantly higher in those cases presenting all features except for positive circumferential and basal margins, and the NSI also had significantly higher values in those cases presenting these features, except for the circumferential and basal margins, as well as the microscopic invasion of the bladder neck. The tumor volume was significantly higher in those cases presenting Gleason score $\geq 7$, Primary Gleason $\geq 4$, intraprostatic perineural invasion and extraprostatic extension.

The measurements of tumor extent were categorized into three groups that were stratified based on 33rd and 66th percentiles as shown in Table 2. For NSI, significant differences between the three categories were observed for all ten morphologic features with prognostic values. For both measurements that were based on the point count method (PGI and tumor volume), significant differences between the three categories were observed for all morphologic features except for the positive basal margins.

\section{Discussion}

The rising incidence of prostate carcinoma and the widespread use of serum PSA screening have resulted in a remarkable increase in the number of radical prostatectomies. Most of these tumors (especially the early diagnosed non-palpable ones) were not grossly detected. Both the quantity of the sampling requirements and the assessments of the quantitative estimation of the tumor volume have considerably increased the workload in many pathology laboratories. In particular, several recommended methods of tumor quantification require specialized tools, such as grids or software, using methods that can be time consuming, such as the demarcation of the tumor by permanent pen directly on the glass slides. The magnitude of effort that pathologists should invest in deriving cancer volume for the pathology reports is still a matter of debate.

Our proposed method assumed that the use of 12 regions of the prostate sample could represent of the entire prostate volume. Several previous reports have suggested that partial embedding methods could generate the same quantification results produced using entire embedding protocols [29]. In a study enrolling 447 patients who underwent prostatectomies, Vollmer concluded that

Table 1

Morphologic features of prostate carcinoma with prognostic value and the associations with three estimates of the tumor extent $(n=143$ ).

\begin{tabular}{|c|c|c|c|c|c|c|}
\hline & \multicolumn{2}{|c|}{ Involved slices $[x / 24]$ mean $\pm \mathrm{SD}$} & \multicolumn{2}{|c|}{$\begin{array}{l}\text { Percentage of prostate } \\
\text { involvement [\%] mean } \pm \text { SD }\end{array}$} & \multicolumn{2}{|c|}{$\begin{array}{l}\text { Tumor volume }\left[\mathrm{cm}^{3}\right] \\
\text { mean } \pm \mathrm{SD}\end{array}$} \\
\hline & Yes & No & Yes & No & Yes & No \\
\hline Gleason score $(\geq 7)$ & $11 \pm 5^{*}$ & $7 \pm 5$ & $21 \pm 12^{*}$ & $10 \pm 10$ & $6 \pm 4^{*}$ & $2 \pm 2$ \\
\hline Primary Gleason ( $\geq 4)$ & $11 \pm 6^{*}$ & $9 \pm 5$ & $26 \pm 14^{*}$ & $13 \pm 10$ & $7 \pm 5^{*}$ & $4 \pm 3$ \\
\hline Intraprostatic perineural invasion & $11 \pm 4^{*}$ & $5 \pm 4$ & $20 \pm 12^{*}$ & $06 \pm 06$ & $5 \pm 4^{*}$ & $2 \pm 2$ \\
\hline Angiolymphatic invasion & $14 \pm 6^{*}$ & $9 \pm 5$ & $28 \pm 11^{*}$ & $14 \pm 12$ & $7 \pm 2$ & $4 \pm 4$ \\
\hline Extraprostatic extension & $13 \pm 5^{*}$ & $9 \pm 5$ & $27 \pm 13^{*}$ & $12 \pm 10$ & $7 \pm 5^{*}$ & $4 \pm 4$ \\
\hline Positive circumferential surgical margin & $11 \pm 4$ & $9 \pm 5$ & $16 \pm 10$ & $14 \pm 12$ & $5 \pm 3$ & $4 \pm 4$ \\
\hline Positive basal surgical margin & $12 \pm 7$ & $9 \pm 5$ & $22 \pm 09$ & $14 \pm 12$ & $6 \pm 5$ & $4 \pm 4$ \\
\hline Positive apical surgical margin & $13 \pm 4^{*}$ & $9 \pm 5$ & $26 \pm 13^{*}$ & $14 \pm 12$ & $5 \pm 2$ & $4 \pm 4$ \\
\hline Seminal vesicle invasion & $13 \pm 5^{*}$ & $9 \pm 5$ & $26 \pm 15^{*}$ & $14 \pm 11$ & $7 \pm 7$ & $4 \pm 4$ \\
\hline
\end{tabular}

\footnotetext{
* Student $t$-test $p<0.05$.
} 


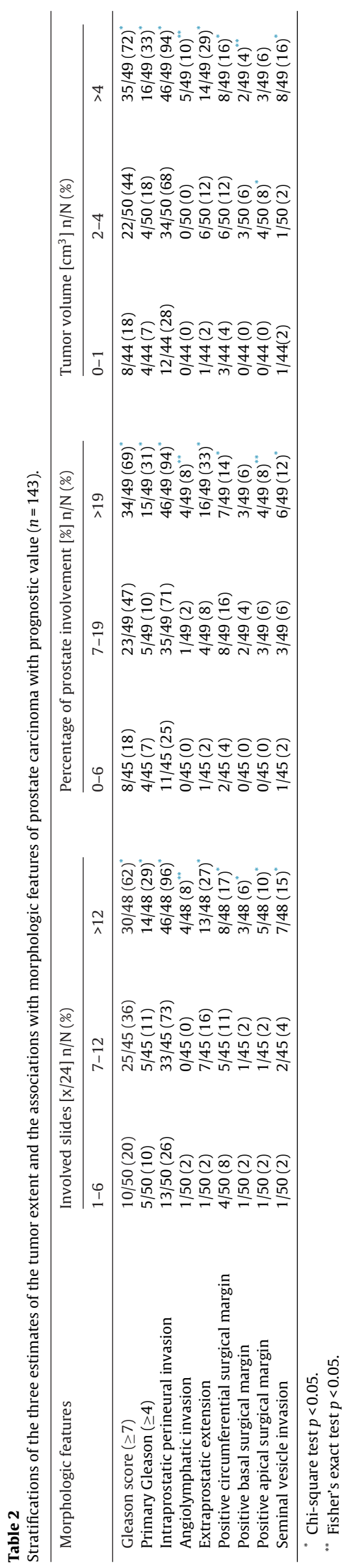

a partial embedding method that was both systematic and unbiased (not preferentially sampling the posterior half of the prostate, for instance) may accurately be used to estimate the percentage of gland involvement by the cancer [31].

Several methods for estimating either the tumor size or the percentage of gland involvement in radical prostatectomy specimens have been used. Three large studies (including 595 and 1850 patients with organ confined disease) suggested that a visual estimation of the percentage of gland involvement (without circling the tumor foci) could be used as an independent factor for the prediction of tumor recurrence [4,22,31]. As demonstrated by Carvalhal and colleagues, each $5 \%$ increase in the percentage of gland involvement was associated with an $11 \%$ increase in disease recurrence [4]. In another report using a visual inspection method, Vollmer reported that, for a series of 447 prostatectomy specimens, the percentage of gland involvement by the cancer was a strong predictor of overall survival (following patient age) that was independent from stage, serum PSA levels and margin status [31]. As visual estimation may be practical for experienced uropathologists, this method may be highly dependent on individual training experiences. The process of circling the complete set of minute tumor foci can be prohibitively time consuming. In another recent report using 645 samples, the percentage of gland involvement (referred to as tumor density) was an independent predictor of cancer recurrence, while tumor volume was not. As a limitation, the prostate tumors were quantified by one of two different methods, including a method that used constructed tumor maps and a method using tumor dimensions with a correction factor derived from the equation for an ellipsoidal structure [17].

Currently, a consensus on the standard method for the partial embedding of prostatectomy specimens has not been established. Epstein and colleagues retrospectively studied 380 radical prostatectomy specimens from T1c (non-palpable, needle biopsy confirmed cancers), presumably containing small tumors that were less likely to be grossly visible. All prostates were embedded in the entirety (mean of 34 slides). Of these samples, 75 cases were selected to include at least one of the adverse findings, such as high Gleason score $(\geq 7)$, positive margins and extraprostatic extension. Nine alternative partial samples strategies were compared with the entire samples to determine the feasibility of detecting these three findings using the partial samples. The strategies were based on extensive sampling of the posterior regions with several alternative methods for the midanterior samplings. The detection of high Gleason scores ranged from $83 \%$ to $98 \%$ in the various methods, with positive margins (71-100\%) and extraprostatic extension (62-96\%) having considerably wider ranges. Based upon these studies, the two recommended methods included a procedure that embedded every posterior section plus one midanterior section of each right and left side (and then all remaining anterior sections of the side if a sizable tumor was detected using microscopy) and a similar method that examined every posterior section that had been obtained only from the ipsilateral areas of previous positive needle biopsies. Using these two strategies, high Gleason scores, positive margins and extraprostatic extensions were detected in $98,100 \%$, and $96 \%$; and $92 \%$; $93 \%$ and $85 \%$, respectively [24]. The asymmetric sampling of the prostate using these methods may possibly induce a potential bias in the calculation of either the tumor volume or the percentage of gland involvement. It is a relevant issue since some estimation of tumor extent is now part of the recommendations for the pathology reports of radical prostatectomy specimens [30].

Other reports have suggested that the partial embedding methods may result in minor misdetections of important features. As reported for a series of 238 specimens by Vainer and colleagues, alternate slicing missed extraprostatic extension in two cases and positive margins in one case [29]. In a series of 148 specimens, Kim and colleagues evaluated five protocols. The rate of detection of 
positive margins and extraprostatic extension was: $83 \%$ and $84 \%$, respectively, for the alternate slicing; $87 \%$ and $88 \%$, respectively, for the alternate slicing with the complete embedding of the biopsypositive posterior quarters; $89 \%$ and $88 \%$, respectively, for every posterior half; $91 \%$ and $90 \%$, respectively, for every posterior sample half plus one midanterior section; and $100 \%$ and $100 \%$, respectively, for the alternate slicing with $3 \mathrm{~mm}$ of the peripheral rim of the remaining prostate. For the fourth method that used the same protocol proposed by Epstein and colleagues (adding the remaining ipsilateral anterior section with the detection of a sizeable tumor) also could allow the detection of all cases of extraprostatic extension and positive margins, with the number of blocks equivalent to entire sampling quantity [16]. Most pathologists would agree that this method is time consuming, requires keeping track of withheld sections and is not suitable for general practice [29]. Recently, a large series of 618 specimens reported discouraging results for the partial sampling methods. The rates of detection of positive margins and extraprostatic extension produced the following results: $87 \%$ and $72 \%$, respectively, for the alternate slices; $79 \%$ and $53 \%$, respectively, for alternate posterior slices plus one midanterior section; and 95\% and 93\%, respectively, for the previously mentioned method recommended by Epstein and colleagues [15].

The main goal of this report was to describe the experience of using a modified point count method to estimate multiple quantitative parameters, such as the tumor volume and the percentage of gland involvement. Consistent with other partial embedding methods, the major limitations of the presented handling protocol include the possibility of underdiagnosing important pathologic features, such as positive margins and extraprostatic extension. After the end of the study period, a combination of the 12 region sampling method (allowing representative sampling of the entire prostate) with the inclusion of the entire peripheral rim of the remaining prostate was used to avoid the potential overlook of positive margins and extraprostatic extensions, as previously discussed in the literature [16].

The proposed method of separating the 12 regions can be readily adopted in pathology laboratories that use either entire embedding or partial embedding with the processing of all peripheral prostate sections. For an average prostate, each one of the 12 regions would result in 4 tissue slices in serial sections. Two alternate slices per region are processed in our protocol. At microscopy, the section with more points counted within the tumor is used to represent the region. The same criterion could apply to the four or more slices in each region using the entire embedding protocols.

Compared with previous recommended protocols, the estimation method of the tumor size described in this study is more practical, generating a procedure that is easy to use by any community-based pathology laboratory and avoiding the use of whole-mounted sections [8], rulers with grid [13], computer-based morphometrics [19] or time consuming drawing on the glass slides or on the paper sheets [3]. After evaluating 447 prostatectomy specimens, Vollmer concluded that the estimation of the percentage of gland involvement by visual inspection would require no more than five minutes. This additional workload would most likely not be a significant issue for reporting this feature. This proposed point count method is well balanced between practicality and reliability, providing a reliable quantitative method that is not dependent only on visual inspection and that could be easily adopted by any practicing pathologist.

The proposed point count method also facilitates the discrimination of the total point count (total tumor percentage or volume) and the specific point count of Gleason 4 and 5 areas (total tumor percentage or volume of high grade prostate carcinoma), a method that has also been suggested as a potential important prognostic marker [5]. The estimation of the amount of high Gleason grade component in each section needed for further evaluation of the average percentage of the entire tumor can be laborious [27], possibly restricting the routine use of this method in the reporting recommendations for pathology reports [9]. After the end of the study period, the percentage of high Gleason grade (4/5) components and the tumor volume of the high grade component of prostate cancer based in the point count method were also included in our reports. An inspection of each region was used to determine the number of the two slides per region that were involved, the number of the possible eight points per region that were involved and the number of points that were involved by high grade $(4 / 5)$ Gleason components. This approach enabled the simple and fast reporting of the total (and high grade) percent of gland involvement by cancer and the tumor volume.

The proposed point count method has several limitations. First, leaving the surgical pathologist to directly draw eight equidistant (or as much as possible equidistant) on the coverslips is subject to some error or adaptation due to variation in size and shape of slices. This limitation is inherent from the original method. Even if primary evaluation could be undisputedly accurate (circling tumor foci), a similar problem of error or adaptation occurs when the pathologist needs to transpose the circled area in perfect quadrant shaped figures. Second, quantification of tumor burden in partially embedded prostates is subject to additional variations. This work was conceived based in the assumption that partial sampling of a prostatectomy specimen may allow estimation of tumor burden if sampling is systematic and not biased from preferentially sampling one region over the other [31]. Small variations in tumor quantification may be related to sampling. In addition, using 12 regions to access percentage of tumor involvement is based on the premise that all regions are symmetrical and with the same volume. The gross examination is the guide to produce 12 regions with equivalent volumes. Indeed, the size and shape of the formalin fixed prostate is somewhat different due to tissue shrinkage (spherical) from the natural anatomy of the prostate in vivo (cone-shaped) [33].

Compared with the various quantitative methods, the use of the ratio of involved slides was considered to be a valid method of tumor size estimation, as this approach correlated moderately with preoperative serum PSA values and was associated with all morphological parameters of prostate cancer progression, except for nodal metastasis. These associations were equivalent for the point count based estimate of the percentage of gland involvement by the carcinoma and the tumor volume. Consistent with other observations in the literature, the use of the number (of percentage) of involved blocks as a measure of the intraglandular extent may be misleading, as a high number of blocks may have small foci of an overall small-sized tumor [25].

\section{Conclusion}

In conclusion, three quantitative methods to estimate the tumor extent were examined, including the number of slices involved by cancer and two methods based on our proposed modified point count method that involved the determinations of the percentage of gland involvement and the tumor volume. The three methods were associated with multiple morphologic features of tumor progression. The two estimates obtained using the modified pointcount method had improved correlations with the preoperative PSA levels.

\section{Conflict of interest}

The authors declare that they have no competing interests. 


\section{Acknowledgments}

This work was supported by a grant from CNPq (National Council for Scientific and Technological Development) 474596/2012-5.

\section{References}

[1] A. Billis, Carcinoma da próstata, in: C.E. Bacchi, M.F. Franco, P.C.C. Almeida (Eds.), Manual de padronização de laudos histopatológicos, 3rd ed., Sociedade Brasileira de Patologia, São Paulo, 2005, pp. 171-185

[2] A. Billis, L.L. Freitas, L.A. Magna, A.B. Samara, U. Ferreira, Prostate cancer with bladder neck involvement: pathologic findings with application of a new practical method for tumor extent evaluation and recurrence-free survival after radical prostatectomy, International Urology and Nephrology 36 (2004) 363-368.

[3] A. Billis, L.A. Magna, U. Ferreira, Correlation between tumor extent in radical prostatectomies and preoperative PSA, histological grade, surgical margins, and extraprostatic extension: application of a new practical method for tumor extent evaluation, International Brazilian Journal of Urology 29 (2003) 113-119.

[4] G.F. Carvalhal, P.A. Humphrey, P. Thorson, Y. Yan, C.G. Ramos, W.J. Catalona, Visual estimate of the percentage of carcinoma is an independent predictor of prostate carcinoma recurrence after radical prostatectomy, Cancer 89 (2000) $1308-1314$

[5] L. Cheng, D.D. Davidson, H. Lin, M.O. Koch, Percentage of Gleason pattern 4 and 5 predicts survival after radical prostatectomy, Cancer 110 (2007) 1967-1972.

[6] T. Dvorak, M.H. Chen, A.A. Renshaw, M. Loffredo, J.P. Richie, A.V. D’Amico, Maximal tumor diameter and the risk of PSA failure in men with specimen-confined prostate cancer, Urology 66 (2005) 1024-1028.

[7] S.N. Eble, S.G. Epstein, I.A. Sesterhenn, Pathology and Genetics of Tumours of the Urinary System and Male Genital Organs, IARC, 2004.

[8] L.E. Eichelberger, M.O. Koch, J.N. Eble, T.M. Ulbright, B.E. Juliar, L. Cheng, Maximum tumor diameter is an independent predictor of prostate-specific antigen recurrence in prostate cancer, Modern Pathology 18 (2005) 886-890.

[9] J.I. Epstein, W.C. Allsbrook Jr., M.B. Amin, L.L. Egevad, I.G. Committee, The 2005 International Society of Urological Pathology (ISUP) consensus conference on Gleason grading of prostatic carcinoma, American Journal of Surgical Pathology 29 (2005) 1228-1242.

[10] J.I. Epstein, M. Carmichael, A.W. Partin, P.C. Walsh, Is tumor volume an independent predictor of progression following radical prostatectomy? A multivariate analysis of 185 clinical stage B adenocarcinomas of the prostate with 5 years of followup, Journal of Urology 149 (1993) 1478-1481.

[11] J.I. Epstein, J.E. Oesterling. P.C. Walsh, Tumor volume versus percentage of specimen involved by tumor correlated with progression in stage A prostatic cancer, Journal of Urology 139 (1988) 980-984.

[12] P.A. Humphrey, Tumor amount in prostatic tissues in relation to patient outcome and management, American Journal of Clinical Pathology 131 (2009) 7-10.

[13] P.A. Humphrey, R.T. Vollmer, Intraglandular tumor extent and prognosis in prostatic carcinoma: application of a grid method to prostatectomy specimens, Human Pathology 21 (1990) 799-804.

[14] INCA, Estimativa 2012 - Incidência de Câncer no Brasil, 2011.

15] V. Iremashvili, S.D. Lokeshwar, M.S. Soloway, L. Pelaez, S.A. Umar, M. Manoharan, M. Jorda, Partial sampling of radical prostatectomy specimens: detection of positive margins and extraprostatic extension, American Journal of Surgical Pathology 37 (2013) 219-225.
[16] K. Kim, P.J. Pak, J.Y. Ro, D. Shin, S.J. Huh, Y.M. Cho, Limited sampling of radical prostatectomy specimens with excellent preservation of prognostic parameters of prostate cancer, Archives of Pathology \& Laboratory Medicine 133 (2009) 1278-1284.

[17] L.T. Lavallee, R.H. Breau, M.A. Preston, G. Raju, C. Morash, S. Doucette, R.G. Gerridzen, J. Eastham, I. Cagiannos, The association between tumour density and prostate cancer recurrence following radical prostatectomy, Canadian Urological Association Journal 5 (2011) 397-401.

[18] J.E. McNeal, R.A. Kindrachuk, F.S. Freiha, D.G. Bostwick, E.A. Redwine, T.A. Stamey, Patterns of progression in prostate cancer, Lancet 327 (1986) 60-63.

[19] M. Noguchi, T.A. Stamey, J.E. McNeal, C.E. Yemoto, Assessment of morphometric measurements of prostate carcinoma volume, Cancer 89 (2000) 1056-1064.

[20] A.W. Partin, J.I. Epstein, K.R. Cho, A.M. Gittelsohn, P.C. Walsh, Morphometric measurement of tumor volume and per cent of gland involvement as predictors of pathological stage in clinical stage B prostate cancer, Journal of Urology 141 (1989) 341-345.

[21] M.M. Quintal, L.A. Magna, M.S. Guimaraes, T. Ruano, U. Ferreira, A. Billis, Prostate cancer pathologic stage pT2b (2002 TNM staging system): does it exist, International Brazilian Journal of Urology 32 (2006) 43-47.

[22] C.G. Ramos, K.A. Roehl, J.A. Antenor, P.A. Humphrey, W.J. Catalona, Percent carcinoma in prostatectomy specimen is associated with risk of recurrence afte radical prostatectomy in patients with pathologically organ confined prostate cancer, Journal of Urology 172 (2004) 137-140.

[23] H. Samaratunga, R. Montironi, L. True, J.I. Epstein, D.F. Griffiths, P.A. Humphrey, T. van der Kwast, T.M. Wheeler, J.R. Srigley, B. Delahunt, L. Egevad, I.P.C. Group, International Society of Urological Pathology (ISUP) consensus conference on handling and staging of radical prostatectomy specimens, Working group 1 : specimen handling, Modern Pathology 24 (2011) 6-15.

[24] A.E. Sehdev, C.C. Pan, J.I. Epstein, Comparative analysis of sampling methods for grossing radical prostatectomy specimens performed for nonpalpable (stage T1c) prostatic adenocarcinoma, Human Pathology 32 (2001) 494-499.

[25] J.R. Srigley, Key issues in handling and reporting radical prostatectomy specimens, Archives of Pathology \& Laboratory Medicine 130 (2006) 303-317.

[26] J.R. Srigley, M.B. Amin, J.I. Epstein, D.J. Grignon, P.A. Humphrey, A.A. Renshaw T.M. Wheeler, C.o.A.P. Members of the Cancer Committee, Updated protocol for the examination of specimens from patients with carcinomas of the prostate gland, Archives of Pathology \& Laboratory Medicine 130 (2006) 936-946.

[27] T.A. Stamey, T.S. Sozen, C.M. Yemoto, J.E. McNeal, Classification of localized untreated prostate cancer based on 791 men treated only with radical prostatectomy: common ground for therapeutic trials and TNM subgroups, Journal of Urology 159 (1998) 2009-2012.

[28] L.D. True, Surgical pathology examination of the prostate gland. Practice survey by American society of clinical pathologists, American Journal of Clinical Pathology 102 (1994) 572-579.

[29] B. Vainer, B.G. Toft, K.E. Olsen, G.K. Jacobsen, N. Marcussen, Handling of radical prostatectomy specimens: total or partial embedding, Histopathology 58 (2011) 211-216

[30] T.H. van der Kwast, M.B. Amin, A. Billis, J.I. Epstein, D. Griffiths, P.A. Humphrey R. Montironi, T.M. Wheeler, J.R. Srigley, L. Egevad, B. Delahunt, I.P.C. Group, International Society of Urological Pathology (ISUP) consensus conference on handling and staging of radical prostatectomy specimens. Working group 2: T2 substaging and prostate cancer volume, Modern Pathology 24 (2011) 16-25.

[31] R.T. Vollmer, Percentage of tumor in prostatectomy specimens: a study of American Veterans, American Journal of Clinical Pathology 131 (2009) 86-91.

[32] WHO, in: P. Boyle, B. Levin (Eds.), World Cancer Report 2008, International Agency for Research on Cancer, Lyon, 2008, pp. 42-43.

[33] S.W. Fine, V.E. Reuter, Anatomy of the prostate revisited: implications for prostate biopsy and zonal origins of prostate cancer, Histopathology 60 (2012) $142-152$ 